\title{
Plantas com potencial medicinal em quintais agroflorestais: diversidade entre comunidades rurais do Portal da Amazônia - Mato Grosso, Brasil
}

\author{
Plants with medicinal potential in agroforestry yards: diversity among rural communities of the
}

Portal da Amazônia - Mato Grosso, Brazil

Plantas con potencial medicinal en patios agroflorestales: diversidad entre comunidades rurales en

Portal da Amazônia - Mato Grosso, Brasil

Recebido: 03/05/2021 | Revisado: 12/05/2021 | Aceito: 27/05/2021 | Publicado: 12/06/2021

\author{
Arildo Gonçalo Pereira \\ ORCID: https://orcid.org/0000-0002-5865-7027 \\ Universidade Federal de São Carlos, Brasil \\ E-mail: arildogp@gmail.com \\ Liliane Cristine Schlemer Alcantara \\ ORCID: https://orcid.org/0000-0001-8502-720X \\ Universidade Federal de Mato Grosso, Brasil \\ E-mail: lilianecsa@yahoo.com.br \\ Renata Evangelista de Oliveira \\ ORCID: https://orcid.org/0000-0002-4410-7809 \\ Universidade Federal de São Carlos, Brasil \\ E-mail: reolivei@ufscar.br \\ Adriana Cavalieri Sais \\ ORCID: https://orcid.org/0000-0002-5169-882X \\ Universidade Federal de São Carlos, Brasil \\ E-mail: acsais@ufscar.br
}

\begin{abstract}
Resumo
Os quintais caracterizam opções de uso da terra para conciliar a produção agrícola e florestal, simultaneamente à conservação dos recursos naturais. Este presente trabalho objetivou caracterizar e identificar as espécies vegetais com potencial medicinal em 44 quintais, implantados e manejados por agricultores familiares, em comunidades rurais nos municípios de Alta Floresta (AF) e Nova Canaã do Norte (NCN), no Portal da Amazônia (MT). Foram feitas visitas guiadas durante o mês de agosto de 2017, a fim de mapear e identificar as espécies presentes. Foram identificadas 151 espécies com potencial medicinal (76 espécies em ambos os municípios, 38 exclusivamente em AF e 37 em NCN, sendo 38\% exóticas e 62\% nativas), pertencentes a 59 famílias botânicas (com destaque a Asteraceae, Lamiaceae, Fabaceae e Euphorbiaceae). As espécies estão distribuídas em três estratos, caracterizando consórcio agroflorestal (42\% são herbáceas, $25 \%$ arbóreas, $23 \%$ arbustivas, $8 \%$ trepadeiras e $1 \%$ epífitas). Os resultados apontam ampla distribuição das espécies e diversificação nos quintais, nas diferentes comunidades, não existindo relação entre a idade/tamanho dos quintais e riqueza de espécies. A escolha de espécies pelos agricultores não segue um padrão, ou seja, a maioria não escolhe as mesmas espécies para o cultivo, e a distribuição dessas espécies em todos os estratos (arbóreo, arbustivo e herbáceo), nos quintais amostrados, indica seu potencial para a construção de consórcios agroflorestais. Destaca-se o elevado número de espécies medicinais do estrato superior, de porte arbóreo, importantes para a reinserção desse componente nas propriedades e comunidades, e na paisagem do Portal, caracterizada por desmatamentos.
\end{abstract}

Palavras-chave: Agricultura familiar; Plantas medicinais; Etnobotânica; Sistemas agroflorestais.

\begin{abstract}
Yards characterize land use options able to reconcile agricultural and forestry production, simultaneously with the conservation of natural resources. This study aimed to characterize and identify plant species with medicinal potential in 44 yards, cultivated and managed by family farmers, in rural communities in Alta Floresta (AF) and Nova Canaã do Norte (NCN), Portal da Amazônia (MT). Guided visits were made in the yards, during the month of August 2017, to map and identify the present species. We identified 151 species with medicinal potential (76 species in both municipalities, 38 exclusively in $\mathrm{AF}$ and 37 in NCN, 38\% exotic and 62\% native), belonging to 59 botanical families (notably Asteraceae, Lamiaceae, Fabaceae and Euphorbiaceae). The species are distributed in three strata, characterizing an agroforestry consortium (42\% are herbaceous, $25 \%$ arboreal, $23 \%$ shrubby, $8 \%$ lianas and vines and $1 \%$ epiphytes). The results point to a wide distribution of species and diversification in yards, in different communities, with no relationship between the age/size of yards and species richness. The choice of species by farmers does not follow a pattern, that is, most do not choose the same species for cultivation, and the distribution of
\end{abstract}


these species in all strata (tree, shrub and herbaceous) in the yards, indicates their potential for the construction of agroforestry consortia. The high number of medicinal species in the upper stratum - of arboreal size - stands out, which is important for the reinsertion of trees in properties, communities, and in the landscape of Portal, characterized by deforestation.

Keywords: Family farming; Medicinal plants; Ethnobotany; Agroforestry systems.

\section{Resumen}

Los patios caracterizan opciones de uso de la tierra para conciliar la producción agrícola y forestal, y conservación de recursos naturales. Apuntamos caracterizar e identificar especies de plantas con potencial medicinal en 44 patios, manejados por agricultores familiares en comunidades rurales de Alta Floresta (AF) y Nova Canaã do Norte (NCN), en Portal da Amazônia (MT). Se realizaron visitas guiadas en agosto de 2017, para mapear y identificar las especies presentes. Se identificaron 151 especies con potencial medicinal (76 en ambos municipios, 38 exclusivamente en AF y 37 en NCN, 38\% exocticas y $62 \%$ nativas), de 59 familias botánicas (destacando Asteraceae, Lamiaceae, Fabaceae y Euphorbiaceae). Las especies se distribuyen en tres estratos, caracterizando consorcio agroforestal (42\% son herbáceas, $25 \%$ arbóreas, $23 \%$ arbustivas, $8 \%$ trepadoras y 1\% epifitas). Los resultados apuntan a una amplia distribución de especies y diversificación en patios, en diferentes comunidades, sin relación entre su edad / tamaño y la riqueza de especies. La elección de especies por los agricultores no sigue un patrón, la mayoría no elige las mismas especies para el cultivo, y la distribución de especies en todos los estratos (arbóreo, arbustivo y herbáceo), en los patios indica su potencial para la construcción de consorcios agroforestales. Se destaca el alto número de especies medicinales en el estrato arbóreo, o que es importantes para la reinserción de este componente en las propiedades y comunidades, y en el paisaje del Portal, caracterizado por la deforestación.

Palabras clave: Agricultura familiar; Plantas medicinales; Etnobotánica; Sistemas agroforestales.

\section{Introdução}

A sociedade humana sempre buscou armazenar conhecimentos a fim de relacionar e organizar as bases fundamentais para sua sobrevivência. Homens e mulheres ainda são, por exemplo, agentes significativos de alteração e transformação da vegetação, modificando-a para atender suas necessidades imediatas e na utilização experimental ou representativa, e atreladas a costumes sociais locais. A relação humana com as plantas é muito rica, relaciona-se a aspectos alimentares (fontes de alimento), estéticos (plantas ornamentais) e de saúde (plantas medicinais), entre outros, e envolve elevada diversidade de grupos socioculturais (Trotta et al. 2012). A escolha das espécies e seus usos por populações humanas estão relacionados a vários fatores, como origem das pessoas, cultura e história de vida, região em que se insere a relação familiar com a natureza, necessidade de subsistência ou complementação da renda, sofrendo influência das expectativas de lazer e ou convivência nos espaços da casa e até mesmo de aspectos ligados à espiritualidade (Almeida \& Gama, 2014).

O Mato Grosso é o terceiro maior estado em extensão brasileiro, possui um território de $903.357,908$ km², com um registro de aproximadamente 118.679 imóveis agrícolas, nos quais mais de 88\% são de agricultura familiar (IBGE, 2017). O Portal da Amazônia, território da cidadania onde se insere esta pesquisa, representa uma região de fronteira agrícola, onde a conversão de áreas de florestas em áreas de cultivo e pecuária extensiva produziu altas taxas de desmatamentos (Cammelli, 2013; Gosch, Ferreira \& Medina, 2017), e onde estão estabelecidos por volta de 16.000 agricultores familiares (Carneiro, Camurça, Esmeraldo \& Sousa, 2010). Também nessa região a implantação de sistemas agroflorestais (SAFs) tem se tornado uma estratégia para subsidiar a adequação legal de propriedades rurais quanto à presença do componente florestal. Dentre as diversas categorias de SAFs, os hortos caseiros mistos ou quintais agroflorestais (QAF) destacam -se como uma das opções viáveis para o aumento da renda e melhoria da segurança alimentar de famílias agricultoras nas paisagens rurais (Bajpai, Sharma \& Kanungo, 2013; Garcia, Vieira \& Oliveira, 2015).

Estudos relativos à segurança alimentar e à agrobiodiversidade conceituaram os quintais como sendo os terrenos localizados ao redor das residências, onde se faz o cultivo ou se conserva múltiplas espécies, que atendem parte das necessidades nutricionais de toda a família, bem como fornecem distintos produtos (Brito \& Coelho, 2000). Para Kumar (2018) essas áreas representam as formas mais clássicas de prática agrícola, desenvolvidas há muitos anos nas regiões tropicais, constituindo associações de plantas - árvores, arbustos, trepadeiras e plantas herbáceas - crescendo próximo às casas. 
São plantados, conservados e manejados pelos membros da família e seus itens são essencialmente para consumo próprio. Diversos autores usam como sinônimos os termos homegardens, pomares agroflorestais, hortos caseiros e quintais agroflorestais (Eichemberg \& Amorozo, 2013; Araújo, Melo \& Albuquerque, 2014; Pasa \& Ávila, 2010). De forma geral, os diversos estudos realizados em torno do tema, retratam esses sistemas como combinações multiestratificadas de árvores e culturas anuais e/ou ainda o uso de animais domésticos, com forte ligação com o domicílio. Apesar de geralmente se constituírem em áreas pequenas, eles fornecem alimentos básicos, plantas medicinais e suprem as necessidades nutricionais das famílias que os cultivam (Chablé-Pascual, et al., 2015).

A estrutura e composição nesses espaços varia amplamente (Gazel-Filho, 2008; Amarozo, 2013; Abebe, Sterck, Wiersum \& Bonger, 2013), dependendo de condições socioeconômicas e ecológicas (Rivas, 2014; Sharma, Baijpai, Shrivastava \& Kanungo, 2014; Zimmerer 2014) e de características culturais relacionadas à história e ao modo de vida de seus manejadores (Plieninger \& Bieling 2013; Idohou et al., 2014; Das \& Das, 2015). A agrobiodiversidade cultivada nesses espaços inclui as mais variadas espécies e hábitos de crescimento (arbóreas, herbáceas, arbustivas, trepadeiras e epífitas) com os mais variados fins (Amorozo, 2013).

O uso e cultivo de plantas consideradas medicinais (aquelas com algum tipo de ação terapêutica) pela população é amplamente difundido (Messias, Menegatto, Prado, Santos \& Guimaraes, 2015), assim como seu cultivo em quintais rurais e urbanos (Adjahossou, Adjahossou, Djego \& Adjahossou, 2016; Neelamegam et al., 2016; Pereira, Jardim, Freitas \& Paraense, 2018). Neste contexto, o presente trabalho objetivou identificar as espécies vegetais com potencial medicinal cultivadas, mantidas e manejadas em quintais agroflorestais e caracterizar esses espaços de cultivo em comunidades rurais de agricultores familiares do Mato Grosso, nos municípios de Alta Floresta e Nova Canaã do Norte.

\section{Material e Métodos}

\subsection{Caracterização da área de estudo}

A pesquisa foi realizada em imóveis rurais localizados em comunidades nos municípios de Alta Floresta e Nova Canaã do Norte, localizados no Portal da Amazônia, no Norte do estado de Mato Grosso (Figura 1). Ambos os municípios foram fundados a partir dos planos de colonização implementados na década de 1970 (Picoli, 2004). Alta Floresta encontra-se a cerca de $800 \mathrm{~km}$ da capital Cuiabá e possui 50.189 habitantes, em área de 8.982,8 km². Nova Cannã do Norte fica a aproximadamente 746,8 Km da capital e possui 12.388 habitantes em uma área de 5.993 km² (INDECO, 2017; IBGE, 2017). 
Figura1 - Localização da região Portal da Amazônia (MT) e dos municípios de Alta Floresta e Nova Canaã do Norte, representados em mosaicos de imagens de satélite.

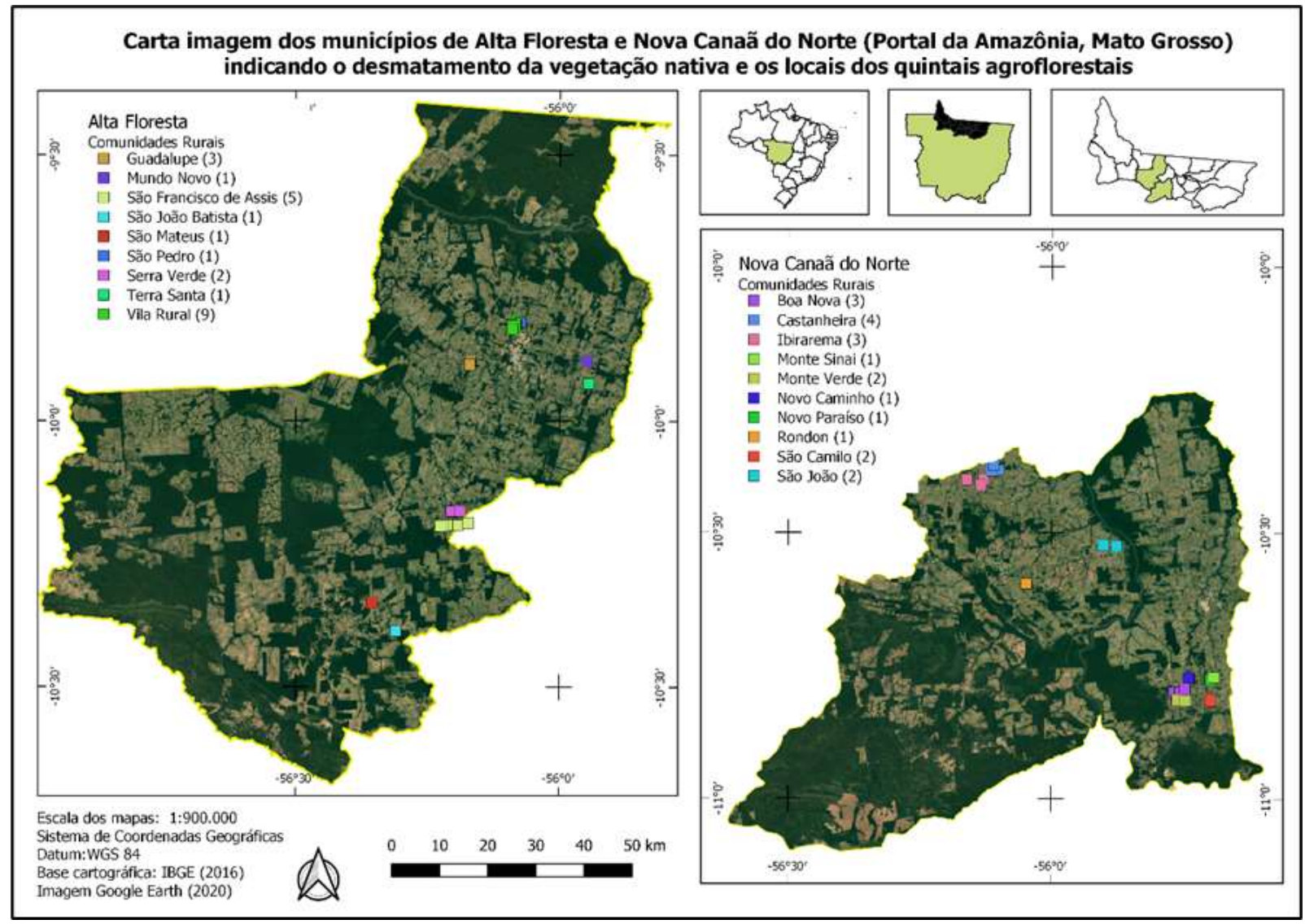

Fonte: Sais (2020).

Na Figura 1, além da localização da área de estudo, os municípios têm como fundo mosaicos de imagens de satélite onde se observa o desmatamento da vegetação nativa (representada em verde escuro). Também foram marcados os 44 quintais analisados, as cores diferentes identificam as 19 comunidades estudadas e número entre parênteses indicam a quantidade de quintais estudados em cada comunidade.

Estes QAF fazem parte do projeto "Sementes do Portal II", idealizado e organizado pela Organização Não Governamental (ONG) Instituto Ouro Verde (IOV), visando atender a demandas de agricultores familiares da região, por sementes e mudas vegetais. As espécies foram implantadas em áreas próximas às casas dos agricultores e agricultoras, principalmente por muvuca, técnica que permite o plantio direto simultâneo de sementes agrícolas e florestais.

\subsection{Procedimentos metodológicos}

A pesquisa classifica-se como descritiva com estudo de caso (Godoy, 1995; Yin, 2001). Foram realizadas visitas guiadas aos quintais, para levantamento e identificação das espécies cultivadas, que ocorreram de 03 a 29 de agosto de 2017. Para a identificação das espécies vegetais, foram levantadas as características morfológicas das plantas e o nome vulgar, confrontando fotografias obtidas in loco com imagens registradas em material bibliográfico fornecido pelo IOV, contendo as espécies implantadas inicialmente nos QAF. Para a correta identificação botânica das espécies foram consultados os trabalhos de Lorenzi e Matos (2008); Guarim Neto e Maciel (2008); Souza e Pasa (2013); Mamede e Pasa (2014). Os nomes científicos das espécies com os respectivos autores foram atualizados posteriormente com consultas às bases Flora do Brasil (2020) e The Plant List (2018). A identificação foi confirmada a partir da comparação morfológica entre as fotos, o material botânico 
(exscicatas) em herbário e consultas bibliográficas (SpeciesLink, 2020). As espécies indicadas pelos agricultores e agricultoras responsáveis pelo manejo dos quintais como de uso medicinal foram incluídas neste levantamento. Também foram inseridas as espécies medicinais constantes no RENISUS 2009 (BRASIL, 2009). Neste primeiro levantamento não foi identificado o uso medicinal específico para cada planta, já que o intuito inicial foi o de avaliar a importância das espécies consideradas pelos agricultores como de potencial medicinal, para a agrobiodiversidade cultivada e na diversificação dos quintais alvo deste estudo.

Técnicas de geoprocessamento, e o sistema de informação geográfica QGIS foram utilizados para a representação espacial da distribuição de espécies. Foram confeccionados mapas para uma análise gráfica da distribuição dos quintais visitados e localização das comunidades rurais nos municípios de Alta Floresta e de Nova Canaã do Norte.

\section{Resultados e Discussão}

\subsection{Perfil dos agricultores}

Por meio dos dados dos agricultores registrados no Instituto Ouro Verde, foi possível avaliar algumas questões referentes ao seu perfil. Em se tratando da idade, houve uma variação entre 23 e 75 anos, sendo que 48,3\% possuem idade inferior a 50 anos e 51,7\% superior a 51 anos de idade. Embora nesta pesquisa não tenha sido objeto de estudo o saber local desses atores sobre plantas medicinais e seus usos, mas sim um levantamento de plantas com potencial medicinal cultivadas nos QAF, cabe ressaltar que já foi observado por diversos estudos etnobotânicos que pessoas mais idosas detêm um maior conhecimento sobre as plantas medicinais (Silva, Junior, Silva \& Silva, 2007; Oliveira \& Cabreira, 2012; Winklerprins \& Souza, 2005; Oliveira et al., 2020). Esse saber já é esperado dessas pessoas, pois tendem a possuir maior conhecimento sobre plantas, em muitos casos, pela própria experiência de frequentemente utilizarem para cuidados com as crianças e demais parentes, vizinhos e amigos.

Notou-se que a maioria dos agricultores são do sexo masculino, sendo que os homens representam $75 \%$ dos agricultores em Alta Floresta e 55\% em Nova Canaã do Norte. Além disso, percebe-se pelos registros que em sua grande maioria, estes agricultores são oriundos de outros estados do país, especialmente da região Sul. Resultados similares foram observados em outros estudos (Barreto \& Spanholi, 2019; Weihs et al., 2020). Dos agricultores participantes do projeto Sementes do Portal II, os registros inventariados pelo IOV indicam que $45 \%$ já praticavam agricultura antes do projeto e outros $55 \%$ possuíam outra profissão, além de que $60 \%$ deles possuem o título de posse da terra e $40 \%$ possuem título de compra e venda.

Nas comunidades visitadas, informações sobre o perfil dos manejadores e suas origens podem trazer insights sobre seu conhecimento e relação com diferentes espécies, já que estas foram selecionadas e introduzidas por opção dos próprios agricultores, fortalecendo, muito provavelmente, as relações entre estes e os recursos vegetais da região (sejam eles nativos ou introduzidos).

\subsection{Caracterização dos quintais}

O tamanho dos quintais agroflorestais é variado: nas áreas estudadas, foram encontrados quintais com superfícies variando de $200 \mathrm{~m}^{2}$ a $20.000 \mathrm{~m}^{2}$. A maioria dos quintais pesquisados é constituída por elevado número de espécies vegetais, que variam desde plantas de hábito herbáceo até arbóreo. Também foram verificadas culturas agrícolas feijão (Phaseolus sp.), abóbora (Curcubita pepo L.), maxixe (Cucumis anguria L.) e mandioca (Manihot esculenta Crantz.) em pequenos espaços (Figura 2). 
Figura 2 - Ilustração dos quintais agroflorestais localizados em diferentes comunidades rurais nos municípios de Alta Floresta (Fig. 2A. Vila Rural; Fig. 2B. Guadalupe) e de Nova Canaã do Norte (Fig. 2C. Serra Verde; Fig. 2D. Novo Paraiso), da região norte do estado de MT, 2017.
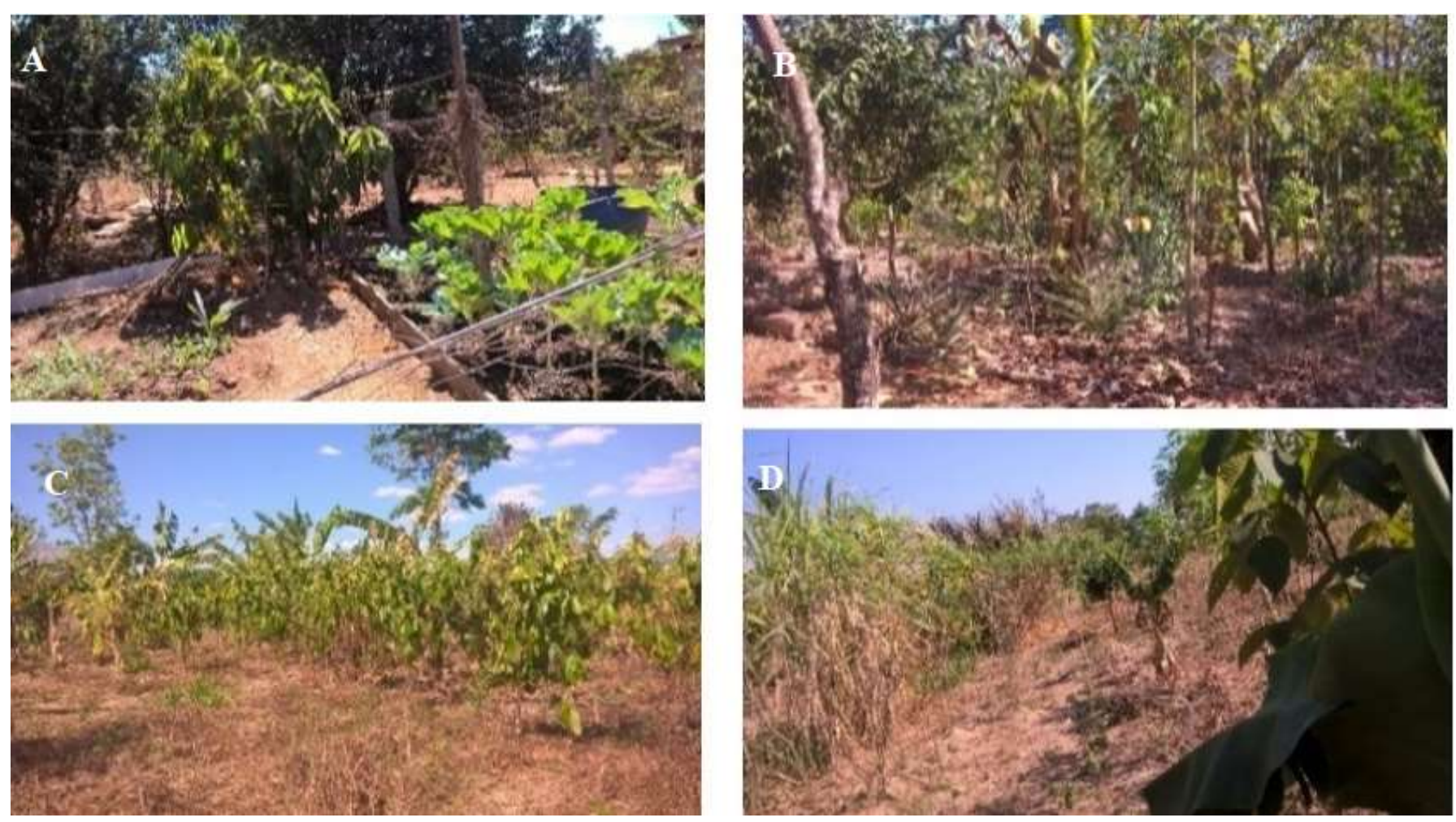

Fonte: Imagem dos arquivos de Pereira, AG.

Na Figura 2 estão quatro fotos de quintais visitados, sendo dois em Alta Floresta e dois em Nova Canaã do Norte. Nessas fotos podem ser verificadas a diversidade de espécies nos estratos vertical e horizontal.

Quanto à estratificação vertical observou-se três estratos. O estrato superior é representado por espécies frutíferas comuns na região, como a mangueira (Mangifera indica L.), coqueiro (Coco nucifera L.), goiabeira (Psidium guajava L.), ainda pode ser encontrado algumas espécies nativas, como ipê roxo (Handroanthus impetiginosus (Mart. ex DC.) Mattos), peroba (Aspidosperma plyneuron Mull. Arg) e guarantã (Esembeckia pumila Pohl.). No estrato intermediário predominam as limeiras (Citrus aurantifolia S.), limoeiros (Citrus limonium Osb.) e acerola (Malpighia glabra L.). O estrato inferior é constituído principalmente, por hortaliças como a cebolinha (Allium schoenoprosum L.), coentro (Coriandrum sativum L.), alface (Lactuca sativa L.), plantas ornamentais e algumas ervas medicinais como: alfazema (Lavandula spica Cav.), babosa (Aloe vera L.), boldo (Peumus boldus), capim santo (Cymbopogon citratus (DC) Stapf.), erva cidreira (Lippia alba (Mill.) N. E. Brown.), camomila (Matricaria recutita L.).

Quanto à estratificação horizontal (alocação e distribuição das espécies nos quintais) constatou-se que não há um padrão de zoneamento para o cultivo das plantas medicinais, ornamentais e de lazer, excetuando-se as hortaliças, que são utilizados na cozinha, geralmente plantadas em locais cercados.

Foram identificadas 151 espécies vegetais, divididas em 62 famílias botânicas, sendo 76 espécies encontradas em ambos os municípios, 38 cultivadas exclusivamente em Alta Floresta e 37 em Nova Canaã do Norte (A lista completa de espécies, organizadas por família botânica, pode ser visualizada na Tabela 1). Esses números mostram uma ampla distribuição de espécies (Bandeira, 2014; Messias et al., 2015). Assim como apontado em estudo realizado por Santos, Alves, Santos, 
Barbosa \& Brasileiro (2012) em comunidade rural do semiárido da Paraíba, o número significativo de espécies de plantas medicinais citadas comprova uma rica distribuição e conhecimento da população local.

Tabela 1 - Lista das espécies com potencial medicinal levantadas em quintais agroflorestais em comunidades rurais nos municípios de Alta Floresta (AF) e Nova Canaã do Norte (NCN), localizados na região norte do estado de MT, 2017, distribuídas por família botânica (Legenda: Os municípios em que as espécies ocorrem, AF (Alta Floresta), NCN (Nova Canaã do Norte). O: origem (E= Exótica; $\mathrm{N}=$ nativa). Em HC (Hábito de crescimento), as siglas indicam o hábito de cada planta $(\mathrm{Hb}=$ herbácea; $\mathrm{Av}=$ arbórea; $\mathrm{Ab}=$ arbustiva; $\mathrm{Tr}=$ trepadeira; $\mathrm{Ep}=$ =pífita).

\begin{tabular}{|c|c|c|c|c|c|}
\hline Espécie & Nome popular & $\mathbf{A F}$ & $\mathrm{NCN}$ & $\mathbf{O}$ & $\mathrm{HC}$ \\
\hline \multicolumn{6}{|l|}{ Acanthaceae } \\
\hline Justicia pectoralis Jacq. & Anador & $\mathrm{x}$ & & $\mathrm{N}$ & $\mathrm{Hb}$ \\
\hline \multicolumn{6}{|l|}{ Adoxaceae } \\
\hline Sambucus nigra $L$. & Sabugueiro & $\mathrm{x}$ & $\mathrm{x}$ & $\mathrm{E}$ & Art \\
\hline \multicolumn{6}{|l|}{ Alismataceae } \\
\hline Echinodorus paniculatus Micheli & Chápeu-de-couro & $\mathrm{x}$ & $\mathrm{x}$ & $\mathrm{N}$ & $\mathrm{Hb}$ \\
\hline \multicolumn{6}{|l|}{ Amaranthaceae } \\
\hline Alternanthera dentata (Moench) S. ex R.E.Fr. & Terramicina & $\mathrm{x}$ & $\mathrm{x}$ & $\mathrm{N}$ & $\mathrm{Hb}$ \\
\hline Amaranthus viridis $\mathrm{L}$. & Caruru & & $\mathrm{x}$ & $\mathrm{E}$ & $\mathrm{Hb}$ \\
\hline Dysphania ambrosioides (L.) Mosyakin \& Clemants & Erva de Santa Maria & $\mathrm{x}$ & $\mathrm{x}$ & $\mathrm{E}$ & $\mathrm{Hb}$ \\
\hline Hebanthe eriantha (Poir.) Pedersen & Fafia & $\mathrm{x}$ & & $\mathrm{N}$ & $\mathrm{Hb}$ \\
\hline \multicolumn{6}{|l|}{ Amaryllidaceae } \\
\hline Allium sativum $\mathrm{L}$. & Alho & & $\mathrm{x}$ & $\mathrm{E}$ & $\mathrm{Hb}$ \\
\hline \multicolumn{6}{|l|}{ Anacardiaceae } \\
\hline Mangifera indica $\mathrm{L}$. & Mangueira & $\mathrm{x}$ & $\mathrm{x}$ & $\mathrm{E}$ & Arb \\
\hline Myracrodruon urundeuva Allemão & Aroeira & $\mathrm{x}$ & & $\mathrm{N}$ & Arb \\
\hline \multicolumn{6}{|l|}{ Annonaceae } \\
\hline Annona muricata L. & Graviola & $\mathrm{x}$ & $\mathrm{x}$ & $\mathrm{E}$ & Arb \\
\hline \multicolumn{6}{|l|}{ Apiaceae } \\
\hline Foeniculum vulgareMill. & Erva doce & & $\mathrm{x}$ & $\mathrm{E}$ & $\mathrm{Hb}$ \\
\hline \multicolumn{6}{|l|}{ Apocynaceae } \\
\hline Aspidosperma carapanauba Pichon & Guarantã & $\mathrm{x}$ & & $\mathrm{N}$ & Arb \\
\hline Aspidosperma polyneuron Mull. Arg & Peroba & $\mathrm{x}$ & & $\mathrm{N}$ & Arb \\
\hline \multicolumn{6}{|l|}{ Araceae } \\
\hline Monstera deliciosa Liebm & Costela de Adão & & $\mathrm{x}$ & $\mathrm{E}$ & $\operatorname{Tr}$ \\
\hline Philodendrom imbe Schott ex. Kunth. & Cipó imbé & & $\mathrm{x}$ & $\mathrm{N}$ & $\operatorname{Tr}$ \\
\hline \multicolumn{6}{|l|}{ Arecaceae } \\
\hline Cocos nucifera $\mathrm{L}$. & Coco & $\mathrm{x}$ & & $\mathrm{E}$ & Arb \\
\hline \multicolumn{6}{|l|}{ Aristolochiaceae } \\
\hline AristolochiaesperanzaeKuntze & Cipó mil homem & $\mathrm{x}$ & $\mathrm{x}$ & $\mathrm{N}$ & $\operatorname{Tr}$ \\
\hline \multicolumn{6}{|l|}{ Asparagaceae } \\
\hline Sansevieria trifasciata Prain & Espada de São Jorge & $\mathrm{x}$ & & $\mathrm{E}$ & $\mathrm{Hb}$ \\
\hline \multicolumn{6}{|l|}{ Asteraceae } \\
\hline Achillea millefolium $\mathrm{L}$. & Mil em folhas & $\mathrm{x}$ & $\mathrm{x}$ & $\mathrm{E}$ & $\mathrm{Hb}$ \\
\hline Achyrocline satureiodes (Lam)DC. & Marcela & & $\mathrm{x}$ & $\mathrm{N}$ & $\mathrm{Hb}$ \\
\hline Ageratum conyzoides L. & Erva de São João & $\mathrm{x}$ & & $\mathrm{N}$ & $\mathrm{Hb}$ \\
\hline Artemisia absithium L. & Losna & $\mathrm{x}$ & $\mathrm{x}$ & $\mathrm{E}$ & $\mathrm{Hb}$ \\
\hline Baccharis genistelloides (Lam). Pers. & Carqueja & $\mathrm{x}$ & $\mathrm{x}$ & $\mathrm{E}$ & $\mathrm{Hb}$ \\
\hline
\end{tabular}


Research, Society and Development, v. 10, n. 6, e59010615713, 2021

(CC BY 4.0) | ISSN 2525-3409 | DOI: http://dx.doi.org/10.33448/rsd-v10i6.15713

\begin{tabular}{|c|c|c|c|c|c|}
\hline Espécie & Nome popular & AF & NCN & $\mathbf{O}$ & $\mathbf{H C}$ \\
\hline Bidens pilosa $\mathrm{L}$. & Picão & $\mathrm{x}$ & $\mathrm{x}$ & $\mathrm{E}$ & $\mathrm{Hb}$ \\
\hline Calendola officinalis $\mathrm{L}$. & Calêndola & $\mathrm{x}$ & & $\mathrm{E}$ & Art \\
\hline Conyza bonariensis (L.) Cronquist & Voadeira & & $\mathrm{x}$ & $\mathrm{N}$ & $\mathrm{Hb}$ \\
\hline Gymnanthemum amygdlinum(Delile) Sch.Bip.ex Walp. & Figatil & $\mathrm{x}$ & $\mathrm{x}$ & $\mathrm{E}$ & Arb \\
\hline Matricaria chamomilla $\mathrm{L}$. & Camomila & $\mathrm{x}$ & $\mathrm{x}$ & $\mathrm{E}$ & $\mathrm{Hb}$ \\
\hline Mikania glomerata Spreng. & Guaco & & $\mathrm{x}$ & $\mathrm{N}$ & $\operatorname{Tr}$ \\
\hline Solidago chilensis Meyen & Arnica & $\mathrm{x}$ & & $\mathrm{N}$ & $\mathrm{Hb}$ \\
\hline Tithonia diversifolia (Hemsl.) A. Gray & Margaridão & $\mathrm{x}$ & $\mathrm{x}$ & $\mathrm{E}$ & $\mathrm{Hb}$ \\
\hline Vernonanthura polyanthes (Sprengel) Vega \& Dematteis & Caferana & & $\mathrm{x}$ & $\mathrm{N}$ & Art \\
\hline Vernonia scabra K. Schum. & Assapeixe & & $\mathrm{x}$ & $\mathrm{E}$ & Art \\
\hline \multicolumn{6}{|l|}{ Bignoniaceae } \\
\hline Crescentia cujete $\mathrm{L}$. & Coité & $\mathrm{x}$ & $\mathrm{x}$ & $\mathrm{E}$ & Arb \\
\hline Fridericia chica (Bonpl.) L.G.Lohmann & Crajiru da Amazônia & $\mathrm{x}$ & & $\mathrm{N}$ & $\operatorname{Tr}$ \\
\hline Handroanthus impetiginosus (Mart. ex DC.) Mattos & Ipê roxo & $\mathrm{x}$ & $\mathrm{x}$ & $\mathrm{N}$ & Arb \\
\hline Jacaranda decurrens Cham & Carobinha & $\mathrm{x}$ & & $\mathrm{N}$ & Arb \\
\hline Tabebuia aurea (Silva Manso) B.\& H. ex S.Moore & Paratudo & $\mathrm{x}$ & & $\mathrm{N}$ & Arb \\
\hline \multicolumn{6}{|l|}{ Bixaceae } \\
\hline Bixa orellana $\mathrm{L}$. & Urucum & $\mathrm{x}$ & $\mathrm{x}$ & $\mathrm{N}$ & Arb \\
\hline Cochlospermum regium (Mart.ex Schrank) Pilg & Algodão do campo & $\mathrm{x}$ & $\mathrm{x}$ & $\mathrm{N}$ & Arb \\
\hline Symphytum officinalle $\mathrm{L}$. & Confrei & $\mathrm{x}$ & $\mathrm{x}$ & $\mathrm{N}$ & $\mathrm{Hb}$ \\
\hline \multicolumn{6}{|l|}{ Brassicaceae } \\
\hline Barbarea vulgaris $\mathrm{R}$. Br. & Erva de Santa Barbara & & $\mathrm{x}$ & $\mathrm{E}$ & $\mathrm{Hb}$ \\
\hline \multicolumn{6}{|l|}{ Bromeliaceae } \\
\hline Ananas comosus (L.) Merril & Abacaxi & & $\mathrm{x}$ & $\mathrm{N}$ & $\mathrm{Hb}$ \\
\hline \multicolumn{6}{|l|}{ Cactaceae } \\
\hline Pereskia aculeata Mill. & Ora pro nobis & & $\mathrm{x}$ & $\mathrm{N}$ & $\operatorname{Tr}$ \\
\hline \multicolumn{6}{|l|}{ Caricaceae } \\
\hline Carica papaya $\mathrm{L}$. & Mamão macho & $\mathrm{x}$ & & $\mathrm{E}$ & Arb \\
\hline \multicolumn{6}{|l|}{ Celastraceae } \\
\hline Monteverdia ilicifolia (Mart. ex Reissek) Biral & Espinheira santa & $\mathrm{x}$ & $\mathrm{x}$ & $\mathrm{N}$ & Arb \\
\hline \multicolumn{6}{|l|}{ Chrysobalanaceae } \\
\hline Chrysobalanus icaco L. & Guajuru & & $\mathrm{x}$ & $\mathrm{N}$ & Art \\
\hline \multicolumn{6}{|l|}{ Convolvulaceae } \\
\hline Ipomoea purga (Wender.) Hayne & Jalapa & $\mathrm{x}$ & & $\mathrm{E}$ & $\operatorname{Tr}$ \\
\hline \multicolumn{6}{|l|}{ Costaceae } \\
\hline Costus spicatus (Jacq.) Sw. & Caninha do brejo & $\mathrm{x}$ & $\mathrm{x}$ & $\mathrm{E}$ & $\mathrm{Hb}$ \\
\hline Costus spiralis (Jacq.) Roscoe & Cana de macaco & $\mathrm{x}$ & $\mathrm{x}$ & $\mathrm{N}$ & $\mathrm{Hb}$ \\
\hline \multicolumn{6}{|l|}{ Crassulaceae } \\
\hline Kalanchoe crenata (Andrews) Haw. & Saião & $\mathrm{x}$ & $\mathrm{x}$ & $\mathrm{E}$ & $\mathrm{Hb}$ \\
\hline Kalanchoe pinnatum (Lam.) DC & Corama & $\mathrm{x}$ & & $\mathrm{E}$ & $\mathrm{Hb}$ \\
\hline \multicolumn{6}{|l|}{ Cupressaceae } \\
\hline Chamaecyparis lawsoniana (A. Murray) Parl. & Cipreste & $\mathrm{x}$ & $\mathrm{x}$ & $\mathrm{E}$ & Art \\
\hline \multicolumn{6}{|l|}{ Curcubitaceae } \\
\hline Momordica charantia $\mathrm{L}$. & Melão de São Caetano & $\mathrm{x}$ & $\mathrm{x}$ & $\mathrm{E}$ & $\operatorname{Tr}$ \\
\hline \multicolumn{6}{|l|}{ Equisetaceae } \\
\hline Equisetum arvense $\mathrm{L}$. & $\begin{array}{c}\text { Cavalinha } \\
8\end{array}$ & $\mathrm{x}$ & & $\mathrm{E}$ & $\mathrm{Hb}$ \\
\hline
\end{tabular}


Research, Society and Development, v. 10, n. 6, e59010615713, 2021

(CC BY 4.0) | ISSN 2525-3409 | DOI: http://dx.doi.org/10.33448/rsd-v10i6.15713

Espécie

\section{Euphorbiaceae}

Croton antisuphilitcus Mart.

Nome popular

AF

NCN

$\mathbf{O}$

HC

Croton cajucara Benth

Canela de perdiz

Sacaca/açacaca

Sangra d'água

Croton urucurana Bail.

Euphorbia hyssopifolhalia L.

Sete sangria

Euphorbia milii Des Moul.

Coroa de cristo

Aveloz

Euphorbia tirucalli $\mathrm{L}$

Cancerosa

Euphorbia umbellata (Pax) Bruyns

Jatropha curcas L.

Pinhão manso

Jatropha gossypiifolia L.

Pinhão roxo

Phyllantus niruri L.

Quebra- pedra

$\begin{array}{cccc}\text { x } & \text { X } & \text { N } & \text { Hb } \\ \text { X } & & \text { N } & \text { Hb } \\ \text { X } & \text { X } & \text { N } & \text { Art } \\ & \text { X } & \text { N } & \text { Hb } \\ \text { X } & & \text { E } & \text { Art } \\ & \text { X } & \text { E } & \text { Art } \\ \text { X } & \text { X } & \text { E } & \text { Art } \\ & \text { X } & \text { E } & \text { Arb } \\ \text { X } & \text { X } & \text { N } & \text { Art } \\ & \text { X } & \text { N } & \text { Hb }\end{array}$

\section{Fabaceae}

Andira anthelmia (Vell) Benth.

Angelim

Bauhinia angulosa Vogel

Bauhinia affinis Vogel

Copaifera langsdorffii Desf.

Dimorphandra mollis Benth

Dipterex odorata (Aubl.) Willd.

Erythrina mulungu Mart.

Hymenaea coubaril Mart.

Leptolobium elegans Vogel

Uncaria tomentosa (Willd. ex Roem. \& Schult.) DC.

Stryphnodendron adstringens (Mart.) Coville

Tamarindus indica $\mathrm{L}$.

Viguieranthus alternans(Benth.) Villiers

Cipó escada de macaco

Pata de vaca

Copaíba

Faveira

Champanhe

Mulungu

Jatobá

Perobinha do campo

Unha de gato

Barbatimão

Tamarindo

Bordão de velho

\begin{tabular}{|c|c|c|}
\hline \multirow[t]{2}{*}{$\mathrm{x}$} & & $\mathrm{N}$ \\
\hline & $\mathrm{X}$ & E \\
\hline \multirow[t]{2}{*}{$X$} & $\mathrm{x}$ & $\mathrm{N}$ \\
\hline & $\mathrm{X}$ & $\mathrm{N}$ \\
\hline $\mathrm{X}$ & & $\mathrm{N}$ \\
\hline $\mathrm{X}$ & $\mathrm{x}$ & $\mathrm{N}$ \\
\hline $\mathrm{X}$ & & $\mathrm{N}$ \\
\hline $\mathrm{X}$ & $\mathrm{x}$ & $\mathrm{N}$ \\
\hline $\mathrm{X}$ & & $\mathrm{N}$ \\
\hline \multirow{3}{*}{$\mathrm{X}$} & $\mathrm{x}$ & $\mathrm{N}$ \\
\hline & & $\mathrm{N}$ \\
\hline & $\mathrm{X}$ & $\mathrm{N}$ \\
\hline X & $\mathrm{x}$ & $\mathrm{N}$ \\
\hline
\end{tabular}

\section{Lamiaceae}

Hyptis paludosa A.St. Hil.ex Benth.

Alevante

Alfazema

Lavandula angustifolia Mill.

Leonotis nepetifolia (L.) R. Br.

Leonurus sibiricus L.

Cordão de frade

Rubim

Mentha arvensis $\mathrm{L}$.

Vick

Mentha piperita L.

Hortelãzinho

Mentha pulegium L.

Poejo

Ocimum basilicum L.

Alfavaca

Origanum minimum L.

Manjericão

Origanum majorana L.

Manjerona

Origanum vulgare L.

Orégano

Plectranthus amboinicus (Lour.) Spreng

Hortelã de folha gorda

Plectrantus barbatus Andr.

Boldo

Plectranthus ornatus Codd.

Boldo chinês

Alecrim

Abacate

Quina 
Research, Society and Development, v. 10, n. 6, e59010615713, 2021

(CC BY 4.0) | ISSN 2525-3409 | DOI: http://dx.doi.org/10.33448/rsd-v10i6.15713

\begin{tabular}{|c|c|c|c|c|c|}
\hline Espécie & Nome popular & $\mathbf{A F}$ & NCN & $\mathbf{O}$ & HC \\
\hline Punica granatum $\mathrm{L}$. & Romã & $\mathrm{x}$ & $\mathrm{x}$ & $\mathrm{E}$ & Art \\
\hline \multicolumn{6}{|l|}{ Malpighiaceae } \\
\hline Malpighia glabra L. & Acerola & $\mathrm{x}$ & $\mathrm{x}$ & $\mathrm{E}$ & Art \\
\hline \multicolumn{6}{|l|}{ Malvaceae } \\
\hline Gossypium herbaceum L. & Algodão & $\mathrm{x}$ & $\mathrm{x}$ & $\mathrm{E}$ & Art \\
\hline Hibiscus acetosella Welw. ex Hiern & Vinagreira roxa & $\mathrm{x}$ & $\mathrm{x}$ & $\mathrm{E}$ & Art \\
\hline Malva sylvestris $\mathrm{L}$. & Malva & & $\mathrm{x}$ & $\mathrm{E}$ & $\mathrm{Hb}$ \\
\hline Ochroma pyramidale (Cav. ex Lam.) Urb. & Bálsamo & $\mathrm{x}$ & $\mathrm{x}$ & $\mathrm{N}$ & Art \\
\hline Sterculia striata A.St. -Hil. \& Naud. & Xixá & $\mathrm{x}$ & $\mathrm{x}$ & $\mathrm{N}$ & Arb \\
\hline \multicolumn{6}{|l|}{ Marantaceae } \\
\hline Maranta arudinaceae $\mathrm{L}$. & Araruta & $\mathrm{x}$ & $\mathrm{x}$ & $\mathrm{N}$ & $\mathrm{Hb}$ \\
\hline \multicolumn{6}{|l|}{ Menispermaceae } \\
\hline Jateorhiza macrantha (Hook.f.) Exell \& Mendonça & Morfina & $\mathrm{x}$ & & $\mathrm{N}$ & $\mathrm{Hb}$ \\
\hline \multicolumn{6}{|l|}{ Moraceae } \\
\hline Maclura tinctoria (L.) D. Don ex Steud. & Moreira & $\mathrm{x}$ & & $\mathrm{N}$ & Art \\
\hline Morus nigra $\mathrm{L}$. & Amora & $\mathrm{x}$ & $\mathrm{x}$ & $\mathrm{N}$ & Arb \\
\hline Pseudolmedia macrophylla Trécul & Flor de paca & & $\mathrm{x}$ & $\mathrm{N}$ & Arb \\
\hline \multicolumn{6}{|l|}{ Moringaceae } \\
\hline Moringa oleifera Lam. & Moringa & $\mathrm{x}$ & $\mathrm{x}$ & $\mathrm{E}$ & $\mathrm{Hb}$ \\
\hline \multicolumn{6}{|l|}{ Myrtaceae } \\
\hline Blepharocalyx salicifoluis (Kunth) O. Berg & Murta & $\mathrm{x}$ & $\mathrm{x}$ & $\mathrm{N}$ & Art \\
\hline Eucalyptus citriodora Hook. & Eucalipto & $\mathrm{x}$ & & $\mathrm{E}$ & Arb \\
\hline Eugenia patrissi Vahl. & Uvaia & $\mathrm{x}$ & & $\mathrm{N}$ & Arb \\
\hline Eugenia uniflora $\mathrm{L}$. & Pitanga & $\mathrm{x}$ & $\mathrm{x}$ & $\mathrm{N}$ & Art \\
\hline Plinia cauliflora (Mart.) Kausel & Jabuticaba & $\mathrm{x}$ & & $\mathrm{N}$ & Arb \\
\hline Psidium guajava $\mathrm{L}$. & Goiaba & $\mathrm{x}$ & $\mathrm{x}$ & $\mathrm{E}$ & Arb \\
\hline Syzygium cumini (L.) Skeels & Jambolão & $\mathrm{x}$ & $\mathrm{x}$ & $\mathrm{E}$ & Arb \\
\hline \multicolumn{6}{|l|}{ Oxalidaceae } \\
\hline Arverrhoa carambola $\mathrm{L}$. & Carambola & & $\mathrm{x}$ & $\mathrm{E}$ & Arb \\
\hline \multicolumn{6}{|l|}{ Passifloraceae } \\
\hline Passiflora edulis Sims. & Maracujá & $\mathrm{x}$ & $\mathrm{x}$ & $\mathrm{N}$ & $\operatorname{Tr}$ \\
\hline \multicolumn{6}{|l|}{ Phytoloccaceae } \\
\hline Petiveria alliacea $\mathrm{L}$. & Guiné & $\mathrm{x}$ & $\mathrm{x}$ & $\mathrm{E}$ & $\mathrm{Hb}$ \\
\hline \multicolumn{6}{|l|}{ Piperaceae } \\
\hline Piper umbellatum(L.) Miq. & Pariparoba & & $\mathrm{x}$ & $\mathrm{N}$ & $\mathrm{Hb}$ \\
\hline \multicolumn{6}{|l|}{ Plantaginaceae } \\
\hline Plantago major $\mathrm{L}$. & Tansagem & & $\mathrm{x}$ & $\mathrm{E}$ & $\mathrm{Hb}$ \\
\hline \multicolumn{6}{|l|}{ Poaceae } \\
\hline Cymbopogon citratus (DC.) Stapt. & Capim cidreira & $\mathrm{x}$ & $\mathrm{x}$ & $\mathrm{E}$ & $\mathrm{Hb}$ \\
\hline Cymbopogon nardus (L.) Rendle & Citronela & $\mathrm{x}$ & $\mathrm{x}$ & $\mathrm{E}$ & $\mathrm{Hb}$ \\
\hline Eleusine indica (L.) Gaertn. & Pé de galinha & & $\mathrm{x}$ & $\mathrm{E}$ & $\mathrm{Hb}$ \\
\hline \multicolumn{6}{|l|}{ Polygonaceae } \\
\hline Polygonum punctatum Elliott & Erva de bicho & $\mathrm{x}$ & & $\mathrm{N}$ & $\mathrm{Hb}$ \\
\hline \multicolumn{6}{|l|}{ Polypodiaceae } \\
\hline Phlebodium decumanum (Willd.) J.Sm. & Rabo de macaco & $\mathrm{x}$ & $\mathrm{x}$ & $\mathrm{N}$ & Ep \\
\hline
\end{tabular}


Research, Society and Development, v. 10, n. 6, e59010615713, 2021

(CC BY 4.0) | ISSN 2525-3409 | DOI: http://dx.doi.org/10.33448/rsd-v10i6.15713

\begin{tabular}{|c|c|c|c|c|c|}
\hline Espécie & Nome popular & $\mathbf{A F}$ & NCN & $\mathbf{O}$ & $\mathrm{HC}$ \\
\hline Portulaca oleracea $\mathrm{L}$. & Beldroega & $\mathrm{x}$ & $\mathrm{x}$ & $\mathrm{E}$ & $\mathrm{Hb}$ \\
\hline \multicolumn{6}{|l|}{ Pteridaceae } \\
\hline Adiantum capills- veneris $\mathrm{L}$. & Avenca & $\mathrm{x}$ & & $\mathrm{E}$ & Ep \\
\hline \multicolumn{6}{|l|}{ Rubiaceae } \\
\hline Genipa americana $\mathrm{L}$. & Jenipapo & $\mathrm{x}$ & & $\mathrm{N}$ & Arb \\
\hline Morinda citrifolia $\mathrm{L}$. & Noni & $\mathrm{x}$ & $\mathrm{x}$ & $\mathrm{E}$ & Arb \\
\hline Psychotria viridis Ruiz \& Pav. & Chacrona & $\mathrm{x}$ & & $\mathrm{N}$ & Art \\
\hline \multicolumn{6}{|l|}{ Rutaceae } \\
\hline Citrus aurantium $\mathrm{L}$. & Laranja & $\mathrm{x}$ & $\mathrm{x}$ & $\mathrm{E}$ & Art \\
\hline Citrus aurantifolia $\mathrm{S}$. & Lima & $\mathrm{x}$ & & $\mathrm{N}$ & Art \\
\hline Citrus limonium Osb. & Limão & $\mathrm{x}$ & $\mathrm{x}$ & $\mathrm{E}$ & Art \\
\hline Ruta graveolens $\mathrm{L}$. & Arruda & $\mathrm{x}$ & $\mathrm{x}$ & $\mathrm{E}$ & $\mathrm{Hb}$ \\
\hline Zanfhoxylum rhoifollium Lam. & Mamica de porca & $\mathrm{x}$ & $\mathrm{x}$ & $\mathrm{N}$ & Arb \\
\hline \multicolumn{6}{|l|}{ Smilacaceae } \\
\hline Smilax irrorata Mart. ex Griseb & Salsa & & $\mathrm{x}$ & $\mathrm{E}$ & $\mathrm{Hb}$ \\
\hline Smilax japicanga Griseb. & Japecangá & & $\mathrm{x}$ & $\mathrm{N}$ & $\mathrm{Hb}$ \\
\hline Smilax oblongifolia Pohl. ex Griseb. & Salsa-parrilha & $\mathrm{x}$ & & $\mathrm{E}$ & $\mathrm{Hb}$ \\
\hline \multicolumn{6}{|l|}{ Solanaceae } \\
\hline Solanum americanum Mill. & Maria preta & & $\mathrm{x}$ & $\mathrm{N}$ & $\mathrm{Hb}$ \\
\hline Solanum paniculatum $L$. & Jurubeba & $\mathrm{x}$ & $\mathrm{x}$ & $\mathrm{N}$ & Art \\
\hline \multicolumn{6}{|l|}{ Urticaceae } \\
\hline Cecropia pachystachya Trécul & Embaúba & $\mathrm{x}$ & $\mathrm{x}$ & $\mathrm{N}$ & Arb \\
\hline Pilea microphylla (L.) Liebm. & Brilhantina & $\mathrm{x}$ & & $\mathrm{E}$ & $\mathrm{Hb}$ \\
\hline Urera baccifera (L.) Gaudich. ex Wedd. & Urtigão brabo & & $\mathrm{x}$ & $\mathrm{N}$ & $\mathrm{Hb}$ \\
\hline \multicolumn{6}{|l|}{ Verbenaceae } \\
\hline Lippia alba (Mill.) N.E.Br. ex P.Wilson & Erva cidreira & $\mathrm{x}$ & $\mathrm{x}$ & $\mathrm{N}$ & $\mathrm{Hb}$ \\
\hline Stachytarpheta cayennensis (Rich.) Vahl & Gervão & $\mathrm{x}$ & $\mathrm{x}$ & $\mathrm{N}$ & Art \\
\hline \multicolumn{6}{|l|}{ Vitaceae } \\
\hline Cissus verticillata $($ L.) Nicolson \& C.E. Jarvis & Insulina & & $\mathrm{x}$ & $\mathrm{N}$ & $\operatorname{Tr}$ \\
\hline \multicolumn{6}{|l|}{ Xanthorrhoeaceae } \\
\hline Aloe vera (L.) Burm & Babosa & $\mathrm{x}$ & $\mathrm{x}$ & $\mathrm{E}$ & $\mathrm{Hb}$ \\
\hline \multicolumn{6}{|l|}{ Zingiberaceae } \\
\hline Curcuma longa $\mathrm{L}$. & Açafrão & $\mathrm{x}$ & $\mathrm{x}$ & $\mathrm{N}$ & $\mathrm{Hb}$ \\
\hline Curcuma zedoaria (Christm.) Roscoe & Zedoária & $\mathrm{x}$ & $\mathrm{x}$ & $\mathrm{N}$ & $\mathrm{Hb}$ \\
\hline Zingiber officinale Roscoe & Gengibre & $\mathrm{x}$ & $\mathrm{x}$ & $\mathrm{E}$ & $\mathrm{Hb}$ \\
\hline
\end{tabular}

Fonte: Elaborado pelo primeiro autor.

A representação espacial da quantidade e da distribuição por hábito das espécies medicinais, assim como sua alocação nos quintais estudados em Alta Floresta e Nova Canaã do Norte seguem apresentadas na Figura 3. 
Figura 3 - Representação espacial da quantidade e da distribuição por hábito das espécies medicinais localizadas nos 24 quintais de comunidades rurais no município de Alta Floresta, MT.
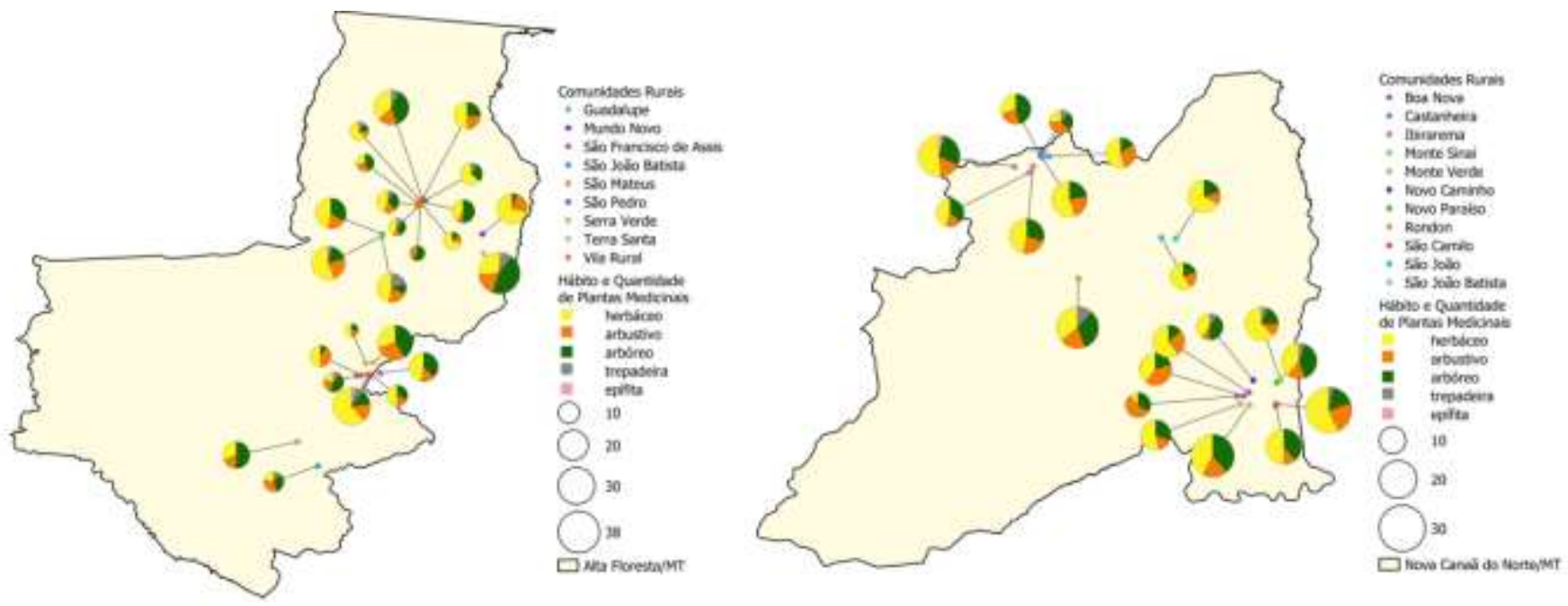

Fonte: Construção dos autores.

A Figura 3 representa os limites municipais de Alta Floresta e Nova Canaã do Norte, onde estão inseridos gráficos em formato de "pizza" de cada quintal visitado. Quanto maior a quantidade de espécies, maior o diâmetro do gráfico. Cores diferentes indicam o hábito dessas plantas (herbáceo, arbustivo, arbóreo, trepadeira ou epífita).

De modo geral, as espécies identificadas apresentam hábito predominantemente herbáceo - 63 (42\%), seguido pelos hábitos arbóreo - 38 (25\%), arbustivo - 35 (23\%), trepadeiras - 13 (8\%) e epífitas - 2 (1\%). Estudos também realizados no estado de Mato Grosso destacam o maior uso medicinal da flora herbácea, seguidas das árvores e arbustos (Añez, 1999; Amaral, 2008; Mamede; \& Pasa, 2018) bem como em outros estados (Amorozo, Pinto \& Furlan, 2006; Fernandez, 2014; Messias et al. 2015). A maior predominância do hábito herbáceo provavelmente está relacionada com uma maior facilidade na coleta e manuseio das plantas.

As famílias com maior representatividade foram Asteraceae e Lamiaceae (15 spp.), Fabaceae (13 spp.), Euphorbiaceae (10 spp.), Myrtaceae (7 spp.). Bignoniaceae, Malvaceae e Rutaceae apresentaram 5 espécies, com números menores de espécies por família nas demais - Amaranthaceae (4 spp.), Bixaceae, Moraceae, Poaceae, Rubiaceae, Smilacaceae, Urticaceae e Zingiberaceae (3 spp.), e as demais famílias com duas ou uma espécie cada. Salienta-se que várias das espécies pertencentes às famílias Asteraceae e Lamiaceae apresentam, segundo a literatura, compostos secundários com ação antimicrobiana e anti-inflamatória, dentre outras funções (Almassy, Lopes, Armond, Silva \& Casali, 2005), com efeitos comprovados por estudos científicos (Lorenzi \& Matos, 2008). Outros estudos também citam as famílias Fabaceae e Lamiaceae como apresentando números significativos de espécies que podem ser usadas como medicinais (Botrel, Rodrigues, Gomes, Carvalho \& Fontes, 2006; Pinto, Amorozo \& Furlan, 2006; Gandolfo \& Hanazaki, 2011).

A estrutura e composição de cada quintal estão associadas, certamente, ao conhecimento e história de vida de cada mantenedor. No levantamento realizado em Alta Floresta e Nova Canaã do Norte, os resultados indicam que os agricultores optam em sua maioria, por espécies herbáceas, cultivadas nas imediações de suas casas. A organização dos componentes que constituem os quintais, como já apontado nesse texto, varia em virtude de questões culturais, espaços, função e história, considerando acontecimentos e práticas vividas. Nos quintais há um rico acervo de recursos vegetais, importante para as famílias que os mantêm, que garantem alimentos, remédios e espaços agradáveis para convivência, além de suporte para uma complexa rede social de trocas de materiais vegetais, especialmente os utilizados na produção alimentícia (IOV, comunicação pessoal). 
Diversos estudos apontam que em quintais, a parte das plantas mais utilizadas para fins medicinais pelos mantenedores é a folha (Jacomassi \& Piedade, 1994; Mosca \& Loiola, 2009; Freitas, Coelho, Maia \& Azevedo, 2012; Bandeira, 2014; Brito, Marín \& Cruz, 2017). Pelos tipos e espécies de plantas levantadas nos quintais visitados, pode-se afirmar que muito provavelmente, seus mantenedores utilizam também a folha em sua maioria. Outros estudos apontam também o uso da casca (Rego, Rocha, Oliveira \& Pacheco, 2016; David \& Pasa, 2015; Santos, Araújo, Sousa \& Lemos, 2016), do fruto, látex e raiz (Trotta et al., 2012; Santos, Salles, Pinto, Pinto \& Rodrigues, 2018), entre outras partes, como o caule, flor, rizoma e sementes (Liporacci \& Simão, 2013; Messias et al., 2015; Rego et al., 2016). O uso das espécies é também diretamente influenciado por sua disponibilidade na região (Duarte, 2001; Campos, 2012; Amaral, 2008; Fernandez, 2014; Macedo \& Ming, 2019) e conhecimentos das pessoas sobre as espécies e seus possíveis usos. Quanto à origem das espécies cultivadas nos quintais, 38\% são exóticas e $62 \%$ são nativas, muitas delas precisando de referências farmacológicas e fitoquímicas para corroborar sua eficiência medicinal.

O número de espécies variou entre as comunidades em Alta Floresta, sendo que o número mais expressivo foi das comunidades Vila Rural e São Francisco de Assis com 79\% e 59\% de espécies respectivamente, em relação as demais. Já em Nova Canaã do Norte não houve grande variação entre as comunidades visitadas; o número de espécies mais expressivo ocorreu nas comunidades Castanheira $43 \%$ e Ibirarema $42 \%$ de espécies respectivamente. Não existe relação entre a idade/tamanho dos quintais e riqueza de espécies (Tabela 2).

Assim como no presente estudo, outros trabalhos apresentaram resultados similares em que, não foi observada relação entre o tamanho dos quintais e riqueza de espécies (Albuquerque, Andrade \& Caballero, 2005; Eichemberg, Amorozo \& Moura, 2009; Aworinde, Erinoso, Ogundairo \& Olanloye, 2013).

Quando se analisa a o número de espécies medicinais por comunidade observa-se aumento nesse número em função do número de quintais visitados em cada comunidade, conforme verificado nos gráficos de dispersão (Figura 4).

Tabela 2 - Número de espécies de plantas com potencial medicinal cultivadas em comunidades rurais dos municípios de Alta Floresta e Nova Canaã do Norte, localizados na região norte do Estado de MT, 2017.

\begin{tabular}{lll}
\hline Comunidades em & $\mathbf{N}^{\mathbf{0}}$ propriedades \\
visitada Floresta - MT & 09 & $\begin{array}{l}\mathbf{N}^{\mathbf{0}} \text { espécies de plantas com } \\
\text { potencial medicinal }\end{array}$ \\
\hline Vila Rural & 03 & 120 \\
Guadalupe & 01 & 57 \\
São Pedro & 01 & 15 \\
Mundo Novo & 05 & 22 \\
São Francisco de Assis & 02 & 89 \\
Serra Verde & 01 & 32 \\
São Mateus (Jacamim) & 01 & 17 \\
São João Batista (Jacamim) & 01 & 16 \\
Terra Santa & & 38 \\
\hline & $\mathbf{N}^{\mathbf{o}}$ propriedades & \\
Comunidades em Nova Canaã do & visitadas & potencial medicinal \\
Norte - MT & 03 & 64 \\
\hline Ibirarema & 04 & 65 \\
Castanheira & 02 & 28 \\
São João Batista & 01 & 25 \\
Rondon & 01 & 20 \\
Novo Paraiso & 01 & 19 \\
Monte Sinai & 02 & 48 \\
São Camilo & 03 & 43 \\
Boa Nova & 01 & 15 \\
Novo Caminho & 02 & 42 \\
Monte Verde & & \\
\hline
\end{tabular}

Fonte: Pesquisa de campo. 
Figura 4 - Gráfico de dispersão entre número de propriedades visitadas por comunidade e número de espécies com potencial medicinal encontradas nos municípios de Alta Floresta, MT $(r=0,97)$ e de Nova Canaã do Norte, MT $(0,91)$.
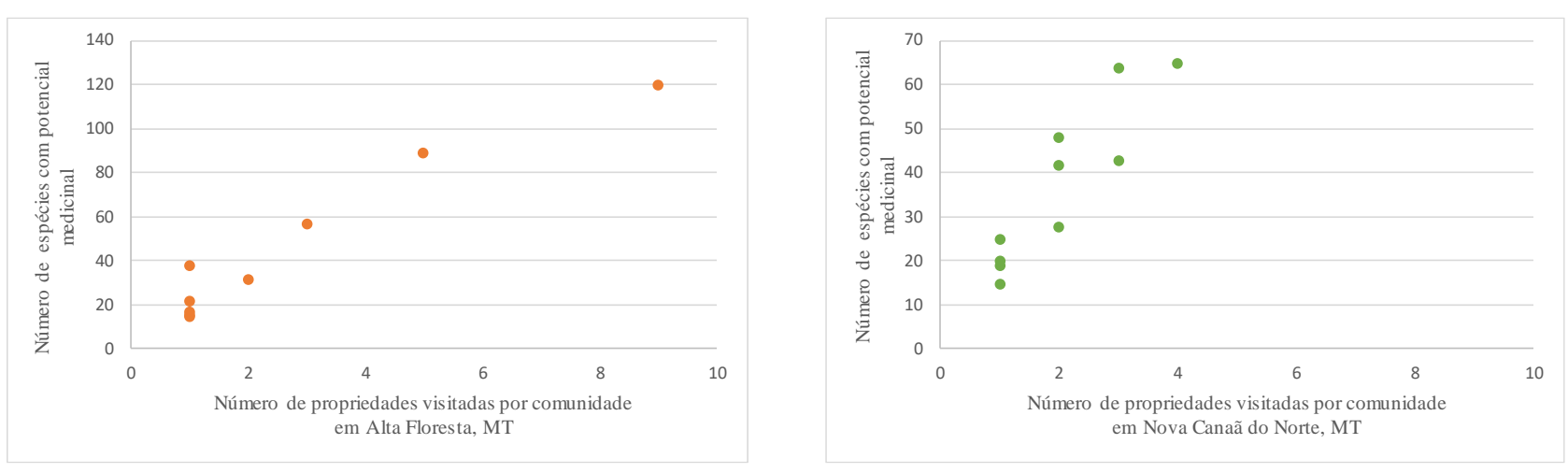

Fonte: Construção dos autores.

A Figura 4 apresenta gráficos de dispersão com correlações muito fortes (de 0,97 para as comunidades de Alta Floresta e de 0,91 para as comunidades de Nova Canaã do Norte) e o aumento é praticamente linear do número de espécies em função do número de quintais amostrados nas comunidades. Isso indica que as escolhas das espécies não seguem um padrão, ou seja, a maioria não escolhe as mesmas espécies para o cultivo.

A distribuição dessas espécies em todos os estratos (arbóreo, arbustivo e herbáceo), nos quintais amostrados, indica seu potencial para a construção de consórcios agroflorestais. Destaca-se o elevado número de espécies medicinais do estrato superior, de porte arbóreo, importantes para a reinserção desse componente nas propriedades e comunidades, e na paisagem.

Devido a sua relação com a saúde e qualidade de vida, as plantas com potencial medicinal, tornam-se justificativas fortes para formar nas comunidades uma percepção da importância de se preservar os recursos naturais, sobre o valor das plantas e a conservação ambiental das espécies nativas. Nas circunstâncias onde o contato com a sociedade de forma geral ou com migrantes aumenta, é provável que aumentem o interesse, tanto de introdução de novas espécies, antes inexistente na região, que são experimentados pela população do local, quanto a novos usos para as espécies já presentes ali. Na medida em que estas plantas e informações sobre elas se disseminam, tende a aumentar o número de pessoas à sua procura.

\section{Considerações Finais}

Frente ao objetivo deste artigo em caracterizar e identificar as espécies vegetais com potencial medicinal em 44 quintais, implantados e manejados por agricultores familiares, em comunidades rurais nos municípios de Alta Floresta (AF) e Nova Canaã do Norte (NCN), no Portal da Amazônia (MT), conclui-se que os quintais possuem um rico acervo de recursos vegetais, importante para a manutenção das famílias, possibilitando o acesso a plantas medicinais, além de alimentos. Além disso, a pesquisa mostrou que plantas medicinais são bastante cultivadas e valorizadas nas comunidades rurais em Alta Floresta e Nova Canaã do Norte, estando presentes em todos os quintais avaliados, o que indica que estas podem estar sendo utilizadas como uma alternativa para o combate e alívio de doenças ou até mesmo como estética e/ou religiosidade pelos agricultores. Consórcios incluindo estas espécies devem ser pensados cuidadosamente, agregando serviços ambientais ao sistema, além de proporcionar outros benefícios aos agricultores.

Este resultado corrobora para demonstrar a importância do cultivo e consequente uso de espécies vegetais com fins medicinais nestas comunidades, agregando valor e diversidade aos chamados quintais agroflorestais. O quintal como objeto deste estudo, tem sua importância destacada enquanto área de socialização rural, palco de cultivo de tradições culturais, produção de conhecimento e como meio de conservação da biodiversidade. 


\section{Agradecimentos}

Este trabalho está integrado ao "Programa de Pesquisa-Ação para avaliação e fortalecimento da Resiliência da Agricultura Familiar no Norte e Noroeste do Mato Grosso". Agradecemos ao Instituto Ouro Verde (IOV) e ao Fundo Amazônia pelo apoio financeiro à realização da Pesquisa, aos técnicos do IOV pelo apoio logístico. Agradecemos ainda aos agricultores e agricultoras que nos permitiram visitar e levantar as espécies cultivadas em suas propriedades.

À Coordenação de Aperfeiçoamento de Pessoal de Nível Superior (CAPES) por concessão de bolsa ao primeiro autor.

\section{Referências}

Abebe T, Sterck F.J, Wiersum K. F, \& Bongers F. (2013). Diversity, composition and density of trees and shrubs in agroforestry homegardens in Southern Ethiopia. AgroforSyst, 87, 1283-1293.

Adjahossou, B. S., Adjahossou, V. N., Djego, J., \& Adjahossou, D. F. (2016). Practice of home gardens (HG) in the Suburban area between Cotonou and Ouidah in Southern Benin. Journal of Biodiversity and Environmental Sciences, 9(5), 29-38.

Albuquerque, U. P., Andrade, L. H. C., \& Caballero, J. (2005). Structure and floristics of homegardens in Northeastern Brazil. Journal of Arid Environments 62, 491-506.

Almassy, J. A. A., Lopes, R. C, Armond, C., Silva, F., \& Casali, V. W. D. (2005). Folhas de Chá: Plantas Medicinais na Terapêutica Humana. Viçosa: Ed. UFV, 233p.

Almeida, L. S. \& Gama, J. R. V. (2014). Quintais agroflorestais: estrutura, composição florística e aspectos socioambientais em área de assentamento rural na Amazônia brasileira. Ciência Florestal, 24(4),1037-1048.

Amaral, C. N. (2008). Recursos vegetais dos tradicionais quintais de Rosário Oeste - Mato Grosso. 2008.80 f. Dissertação (Mestrado em Ecologia e Conservação da Biodiversidade) - Universidade Federal de Mato Grosso, Cuiabá.

Amorozo, M. C. M., Pinto, E. P. P., \& Furlan, A. (2006). Conhecimento popular sobre plantas medicinais em comunidades rurais de mata atlântica - Itacaré, BA, Brasil. Acta Botânica Brasílica, 20(4), 751-762.

Amorozo, M. C. M. (2013). Perspectivas atuais para a conservação on farm da agrobiodiversidade. In: Sistemas agrícolas de pequena escala e a manutenção da agrobiodiversidade: uma revisão e contribuições. Botucatu, SP:FCA-Unesp, 97-120.

Añez, R. B. S. (1999). O uso de plantas medicinais na comunidade do Garcês. 157f. Dissertação (Mestrado em Saúde Coletiva) - Universidade Federal de Mato Grosso, Cuiabá-MT.

Araújo, T. A. S., Melo, J. G. \& Albuquerque, U. P. (2014). Plantas medicinais. In: Albuquerque, U.P. (org.) Introdução à etnobiologia. Recife: NUPEEA. 9198.

Aworinde, D. O., Erinoso, S. M., Ogundairo B. O., \& Olanloye, A. O. (2013). Assessment of plants grown and maintained in home gardens in Odeda area Southwestern Nigeria. Journal of Horticulture and Forestry 5, 29-36.

Barreto, M. R., \& Spanholi, M. L. (2019). Estudo etnobotânico em comunidades rurais de Sinop, Mato Grosso, Brasil. Interações, $20(1), 267-282$.

Bajpai, S., Sharma, A. K., \& Kanungo, V. K. (2013). Traditional home gardens: A preserve of medicinal plants. International Journal of Herbal Medicine, v.1, n.2, 152-161.

Bandeira, A. S. (2014). Etnoconhecimento da utilização de plantas medicinais na $14^{a}$ região administrativa da emater-PB. Dissertação (Mestrado em Sistemas Agroindustriais - PPGSA). UFCG, 60f.

Botrel, R. T., Rodrigues, L. A., Gomes, L. J., Carvalho, D. A., \& Fontes, M. A. L. (2006). Uso da vegetação nativa pela população local no município de Ingaí, MG, Brasil. Acta Botanica Brasílica v. 20, 143-156.

Brasil. (2009). Relação Nacional de Plantas Medicinais de Interesse ao SUS. https://portalarquivos2.saude.gov.br/images/pdf/2014/maio/07/renisus.pdf.

Brito, M. A., \& Coelho, M. F. B. (2000). Os quintais agroflorestais em regiões tropicais - unidades autossustentáveis. Agricultura Tropical, Cuiabá, v.4, n.1, 7- 38 .

Brito, M. F. M., Marín, E. A, \& Cruz, D. D. (2017). Plantas Medicinais nos Assentamentos Rurais em uma Área de Proteção no Litoral do Nordeste Brasileiro. Ambiente \& Sociedade. XX(1), 83-104.

Cammelli, F. (2013). Smallholders' collective action and fire risk in the Brazilian Amazon. 133 f. Dissertação (Mestrado em Desenvolvimento Econômico) Scuola di Economia e Management, Università Degli Studi Firenze, Firenze.

Campos, P. A. (2012). Nobres quintais: uma abordagem etnoecológia dos recursos vegetais cultivados nos quintais urbanos de Nobres - Mato Grosso, Brasil. 58f. Dissertação (Mestrado em Ecologia e conservação da biodiversidade) - Universidade Federal de Mato Grosso, Ppg - IB-UFMT, Cuiabá.

Carneiro, M. G. R., Camurça, A. M., Esmeraldo, G. G. S. L. \& Sousa, N. R. (2010). Quintais Produtivos: contribuição Conselho Executivo de Ações da Agricultura Familiar (CEAAF). Plano Territorial de Desenvolvimento Rural Sustentável. Território da Cidadania Portal da Amazônia. Alta Floresta - MT. 2010. http://sit.mda.gov.br/download/ptdrs/ptdrs_qua_territorio074.pdf. 
Chablé-Pascual, R., Palma-Lopez, D. J., Vazquez-Navarrete, C. J., Ruiz-Rosado, O., Mariaca-Mendez, R., \& Ascensio-Rivera, J. M. (2015). Estructura, diversidad y uso de las especies en huertos familiares de la Chontalpa, Tabasco, México. Ecositemas y Recursos Agropecuarios. 2 (4), 23-39.

Das T., \& Das A. K. (2015) Conservation of plant diversity in rural homegardens with cultural and geographical variation in three districts of Barak Valley, Northeast India. Econ Bot 69, 57-71.

David, M., \& Pasa, M. C. (2015). As plantas medicinais e a etnobotânica em Várzea Grande, MT, Brasil. Interações, 16(1), 97-108.

Duarte, T. G. (2001). Um estudo etnoceológico sobre o uso de Recursos Vegetais em Nova Xavantina, Mato Grosso, Cuiabá, MT. (Dissertação de Mestrado). Instituto de Biociências/ UFMT. 135 p.

Eichemberg, M. T., Amorozo, M. C. M., \& Moura, L. C. (2009). Species composition and plant use in old urban homegardens in Rio Claro, Southeast of Brazil. Acta Botanica Brasilica, 23, 1057-1075.

Eichemberg, M. T., \& Amorozo, M. C. M. (2013). Contributions of the old urban homegardens for food production and consumption in Rio Claro, Southeastern Brazil. Boletim do Museu Paraense Emílio Goeldi. Ciências Humanas, 8(3), 745-755.

Fernandez, P. (2014). Plantas medicinais: conhecimento e uso nos espaços rurais do planalto sul catarinense. Tese (Doutorado em Produção Vegetal) Universidade do Estado de Santa Catarina, Lages, $152 \mathrm{p}$

Flora do Brasil (2020). Lista de Espécies da Flora do Brasil do Jardim Botânico do Rio de Janeiro 2020. < http://floradobrasil.jbrj.gov.br >.

Freitas, A. V. L., Coelho, M. F. B., Maia, S. S. S., \& Azevedo, R. A. B. (2012). Plantas medicinais: um estudo etnobotânico nos quintais do Sítio Cruz, São Miguel, Rio Grande do Norte, Brasil. Revista Brasileira de Biociências, 10(1), 48-59.

Gandolfo, E. S., \& Hanazaki, N. (2011). Etnobotânica e urbanização: conhecimento e utilização de plantas de restinga pela comunidade nativa do distrito do Campeche (Florianópolis, SC). Acta Botanica Brasilica, 25, 168-177.

Garcia, B. N. R., Vieira, T. A., \& Oliveira, F. A. (2015). Quintais agroflorestais e segurança alimentar em uma comunidade rural na Amazônia Oriental. Rev. Fac. Agron. La Plata. 114(1), 67-73.

Gazel-filho, A. B. (2008). Composição, estrutura e função de quintais agroflorestais no Município de Mazagão, Belém, 2008.104 f. Tese (Doutorado em Ciências Agrárias). Universidade Federal Rural da Amazônia, Embrapa Amazônia Oriental, Belém.

Godoy, A. S. (1995). Pesquisa qualitativa- tipos fundamentais. Revista de Administração de empresas, 35 (3), 20 -29.

Gosch, M. S., Ferreira, M. E., \& Medina, G. S. (2017). The role of the rural settlements in the Brazilian savanna deforestation process. Journal of Land Use Science, 12, 55-70. 10.1080/1747423X.2016.1254687

Guarim Neto, G., \& Maciel, M.R.A. (2008). O saber local e os recursos vegetais em Juruena Mato Grosso, Brasil.

Instituto Brasileiro de Geografia e Estatística (IBGE). (2017). Censo Agropecuário 2017: resultados definitivos. https://censos.ibge.gov.br/agro/2017/resultados-censo-agro-2017/resultados-definitivos.html.

Instituto Brasileiro de Geografia e Estatística (IBGE). (2017). Cidades - Mato Grosso - Alta Floresta e Nova Canaã do Norte. 2017. <hpp:/l cidades.ibge.gov.br/brasil/mt/alta-floresta/panorama/> e < hpp:// cidades.ibge.gov.br/brasil/mt/nova-canaa-do-norte/panorama .

Idohou R., Fandohan B., Salako V., Kassa B., Gbedomon R.C., Yedomonhan H., Glele Kakai R.L., \& Assogbadjo A.E. (2014). Biodiversity conservation in home gardens: traditional knowledge, use patterns and implications for management. Int J Biodiv Sci Ecosyst Serv Manag 10, 89-100.

INDECO - Integração, Desenvolvimento e Colonização. (2017). http://www.colonizadoraindeco.com.br/altafloresta.html.

Jacomassi, E., \& Piedade, L. H. (1994). A importância das plantas com finalidades terapêuticas e suas aplicações na cidade de Goioerê - PR. Revista UNIMAR, 16(2), 335-353.

Kumar, V. (2018). Agrobiodiversity, Structural Compositions and Species Utilization of Homegardens in Humid Tropics, Kerala, India. Tropical Ecology.

Liporacci, H. S. N., \& Simão, D. G. (2013). Levantamento etnobotânico de plantas medicinais nos quintais do Bairro Novo Horizonte, Ituiutaba, MG. Revista Brasileira de Plantas Medicinais, 15(4), 529-540. http://dx.doi.org/10.1590/S1516-05722013000400009.

Lorenzi, H., \& Matos, F. J. A. (2008). Plantas medicinais no Brasil: nativas e exóticas. (2a ed.), Instituto Plantarum, 544 p.

Macedo, G.S. S. R., \& Ming, L. C. (2019). Plantas alimentícias e paisagens: uso e conservação no Sertão do Ubatumirim, Ubatuba, Brasil. Desenvolv. Meio Ambiente, v. 52, dezembro, 194-216.

Mamede, J. S. S., \& Pasa, M. C. (2018). Plantas medicinais utilizadas pela comunidade São Miguel, zona rural de Várzea Grande, Mato Grosso, Brasil. Flovet. 1, n. $6,2014$.

Mattsson, E., Ostwald, M., Nissanka, S. P., \& Marambe, B. (2013). Homegardns as a multifunctional land use strategy in Sri Lanka with a focus on carbon sequestration. AMBIO, 42, 892-902.

Messias, M. C. T. B., Menegatto, M. F., Prado, A. C. C., Santos, B. R., \& Guimarães, M. F. M. (2015). Uso popular de plantas medicinais e perfil socioeconômico dos usuários: um estudo em área urbana em Ouro Preto, MG, Brasil.Rev. Bras. Pl. Med., Campinas, v.17, n.1, 76-104.

Mosca, V. P., \& Loiola, M. I. B. (2009). Uso popular de plantas medicinais no Rio Grande do Norte, nordeste do Brasil. Revista Caatinga, Mossoró, v.22, n., 225-234. 
Neelamegan, R., Muthu, B., Ancy, E. S., Ramani, M., Sindhu, Prathiba, N., Thangasutha, G., \& Mohammed Irsth, J. (2016). Household economy and homegarden plants composition, diversity and utilization in rural villages of Kanyakumari District, Tamil Nadu, India. International Journal of Applied and Pure Science and Agriculture (IJAPSA), 2(1), 12-24.

Oliveira Júnior, C. J. F., \& Cabreira, P. P. (2012). Sistemas agroflorestais: potencial econômico da biodiversidade vegetal a partir do conhecimento tradicional ou local. Revista Verde (Mossoró - RN - Brasil), 7(1), 212224, janeiro-março.

Oliveira, F. R. A., Araujo, R. A. F., Nicacio, R. A. R., Pinto, G. F., Mattos, M., Santos, D. A. S., Olinda, R. A., \& Goulart, L. S. (2020). Prevalência e fatores associados ao uso de plantas medicinais em Rondonópolis, Mato Grosso, Brasil. Revista Enfermagem Atual in Derme, $92-30$.

Pasa, M. C. \& Ávila, G. (2010). Ribeirinhos e recursos vegetais: a etnobotânica em Rondonópolis, Mato Grosso, Brasil. Interações, 11(2), 195-204. http://www.interacoes. ucdb.br/article/view/366.

Pereira, S. C. B., Jardim, I. N., Freitas, A. D. D., \& Paraense, V. C. (2018). Levantamento Etnobotânico de Quintais Agroflorestais em Agrovila no Município de Altamira, Pará. Revista Verde de Agroecologia e Desenvolvimento Sustentável, 13(2), 200-207.

Picoli, F. (2004). Amazônia: do sangue ao mel. Os extremos da expansão capitalista. Sinop: Ed. Amazônia, 2004. 120p.

Pinto, E. P. P., Amorozo, M. C. M., \& Furlan, A. (2006). Conhecimento popular sobre plantas medicinais em comunidades rurais de mata atlântica - Itacaré, BA, Brasil. Acta Botanica Brasilica. 20, 751-762.

Plieninger T., \& Bieling C. (2013). Resilience-based perspectives to guiding high-nature-value farmland through socioeconomic change. Ecol Soc 18(4):20. doi.org./10.5751/ES-05877-180420

Rego, C. A. R. M., Rocha, A. E., Oliveira, C. A., \& Pacheco, F. P. F. (2016). Levantamento etnobotânico em comunidade tradicional do assentamento Pedra Suada, do município de Cachoeira Grande, Maranhão, Brasil. Acta Agron. 65 (3) 284-291.

Rivas, G. (2014). Huertos familiares para la conservación de la agrobiodiversidad, la promoción de la seguridad alimentaria y la adaptación al cambio climático. Ambiéntico, 243, 4-9.

Sais, A. C. (2020). Carta imagem dos municípios de Alta Floresta e Nova Canaã do Norte, Portal da Amazônia, Mato Grosso, Brasil com representação de comunidades e imóveis rurais que possuem quintais agroflorestais. Araras: UFSCar, mapa: 21 x 29,7 cm. Escala: 900.000 1:800:000 Escala 1:600.000.

Santos, S. L. D. X., Alves, R. N., Santos, S. L. D. X., \& Barbosa, J. A. A., \& Brasileiro, T. F. (2012). Plantas utilizadas como medicinais em uma comunidade rural do semiárido da Paraíba, Nordeste do Brasil. Revista Brasileira de Farmácia, 93 68-79.

Santos, A. B. N., Araújo, M. P., Sousa, R. S., \& Lemos, J. R. (2016). Plantas medicinais conhecidas na zona urbana de Cajueiro da Praia, Piauí, Nordeste do Brasil. Rev. Bras. Pl. Med., 18(2), 442-450.

Santos, L. S. N., Salles, M. G. F., Pinto, C. M., Pinto, O. R. O., \& Rodrigues, I. C. S. (2018). O saber etnobotânico sobre plantas medicinais na comunidade da Brenha, Redenção, CE. Agrarian Academy, Centro Científico Conhecer 5(9); 409-421.

Sharma, A. K., Baijpai, S., Shrivastava, S., \& Kanungo, V. K. (2014). Inventorying medicinal plants in urban homegardens of Raipur, Chhattisgarh. International Journal of Herbal Medicine, 2 (1), 43-50.

Silva, M. P. L., Junior, A. A. A., Silva, da F., \& Silva; da M. (2007). Levantamento etnobotânico e etnofarmacológico de plantas medicinais utilizadas por comunidades rurais de Mutuipe-Ba integrantes do "projeto ervas". Cruz das Almas - BA.

Souza, M. D., \& Pasa, M.C. (2013). Levantamento etnobotânico de plantas medicinais em uma área rural na região de Rondonópolis, Mato Grosso. Biodiversidade, 12(1), 138-45.

SpeciesLink. (2020). <http//splink.cri.org.br/>.

The Plant List: A working list of all plant species. Version (2018). <http://www. theplantlist.org>.

Trotta J., Messias, P.A., Pires, A. H. C., Hayashida, C. T., Camargo, C., \& Futemma, C. (2012). Análise do conhecimento e uso popular de plantas de quintais urbanos no estado de São Paulo, Brasil. REA - Revista de Estudos Ambientais, 14(3), 17-34.

Yin, R. K. (2001). Estudo de caso: planejamento e métodos / Robert K. Yin; trad. Daniel Grassi. (2a ed.), Bookman.

Weihs, M., Lopes, F. J. A., Cardoso, S. M. C., Camargo, A. C., Silva, F. O., \& Ruedell, C. M. (2020). Implicações do modelo de ocupação da fronteira agrícola à agricultura familiar em Terra Nova do Norte e Nova Guarita, Amazônia mato-grossense. Desenvolv. Meio Ambiente, 54, 66-84.

Winklerprins, A. M. G. A., \& Souza, P. S. (2005). Surviving the City: Urban Home Gardens and the Economy of Affection in the Brazilian Amazon. Journal of Latin American geography, 4(1), 107-126.

Zimmerer K. S. (2014). Conserving agrobiodiversity amid global change, migration, and nontraditional livelihood networks: the dynamic uses of cultural landscape knowledge. Ecol Soc 19. 1-15. http://dx.doi.org/10.5751/ES-06316-190201. 